\title{
The Experience of Homebound Older Adults During the COVID-19 Pandemic
}

\author{
Claire K. Ankuda, MD, MPH ${ }^{7}$ (D), Ashwin Kotwal2,3, Jennifer Reckrey, MD', \\ Krista L. Harrison, $\mathrm{PhD}^{2,4}$, and Katherine A. Ornstein, $\mathrm{PhD}, \mathrm{MPH}^{1}$
}

'Brookdale Department of Geriatrics and Palliative Medicine, Icahn School of Medicine, New York, NY, USA; ${ }^{2}$ Division of Geriatrics, Department of Medicine, University of California San Francisco, San Francisco, CA, USA; ${ }^{3}$ San Francisco Veterans Affairs Medical Center, San Francisco, CA, USA; ${ }^{4}$ Philip R. Lee Institute for Health Policy Studies, University of California San Francisco, San Francisco, CA, USA.

BACKGROUND: Homebound older adults have heightened risks for isolation and negative health consequences, but it is unclear how COVID-19 has impacted them. We examine social contact and mood symptoms among previously homebound older adults during the COVID-19 pandemic.

DESIGN/SETTING: Cross-sectional analysis using data from the National Health and Aging Trends Study (NHATS), a nationally-representative longitudinal study of aging in the USA.

PARTICIPANTS: A total of 3,112 community-dwelling older adults in 2019 who completed the COVID-19 survey in the summer/fall of 2020.

MEASUREMENTS: Homebound status was defined via self-report as rarely/never leaving home or leaving the house with difficulty or help in the prior month. We measured limited social contact during COVID-19 (in-person, telephone, video or email contacts <once/week), as well as loneliness, anxiety, and depression.

RESULTS: Among homebound older adults, 13.2\% experienced limited social contact during COVID-19 vs. $6.5 \%$ of the non-homebound. Differences in social contact were greatest for contacts via email/text/social media: $54.9 \%$ of the homebound used this <once/week vs. $28.4 \%$ of the non-homebound. In adjusted analyses of those without limited social contact prior to the pandemic, the homebound had higher but not significantly different odds (OR 1.83; 95\% CI 0.95-3.52) of limited social contact during COVID-19, with increased risk among the older individuals, those with dementia, and those in assisted living facilities. Of the homebound, 13.2\% felt lonely every/ most days during the pandemic vs. $7.7 \%$ of nonhomebound older adults. Homebound and nonhomebound older adults reported similar rates of increased loneliness, anxiety, or depression during COVID-19. Fewer homebound older adults learned a new technology during the pandemic (16.3\%) vs. nonhomebound older adults (30.4\%).

DISCUSSION: Isolation among homebound older adults increased during COVID-19, partially due to differences in technology use. We must ensure that homebound persons have the connection and care they need including new technologies for communication during and beyond COVID-19.

Received May 26, 2021

Accepted December 16, 2021

Published online February 15, 2022
KEY WORDS: Homebound; COVID-19; NHATS; Isolation; Social contact.

J Gen Intern Med 37(5):1177-82

DOI: $10.1007 / \mathrm{s} 11606-021-07361-9$

(C) The Author(s) under exclusive licence to Society of General Internal Medicine 2022

\section{BACKGROUND}

More than 2 million older adults in the USA are homebound, or rarely/never leaving home, with over 5 million leaving home only with difficulty or relying on others' assistance. ${ }^{1,2}$ Homebound older adults experience a high symptom burden, reporting greater levels of pain and dyspnea, and significant functional impairment. ${ }^{3}$ They have high levels of mortality, even compared to counterparts with the same demographic, functional, and clinical characteristics. ${ }^{4,5}$ The greater levels of illness experienced by homebound older adults are evidenced by disproportionately high rates of hospital and emergency department utilization. ${ }^{6,7}$

Homebound older adults are also particularly vulnerable to social isolation and its psychological sequelae. Social isolation, defined as reduced engagement and minimal contact with others, has been associated with multiple adverse health impacts. ${ }^{8}$ Indeed, homebound older adults have demonstrated higher levels of depression than their non-homebound counterparts. 9,10

Little is known about how the coronavirus-19(COVID-19) pandemic has impacted homebound older adults. Given their high degree of functional disability and comorbidities, homebound older adults are at high risk of serious complications or death. ${ }^{11}$ They are also more likely to experience disruptions in medical care and caregiving due to the pandemic; for example, the strain of the pandemic was noted both on formal homebased medical care services as well as home health aides. ${ }^{12,13}$ This is similar to research in other disasters, demonstrating that homebound older adults are more likely to face challenges meeting their care and medical needs during and after events such as floods and earthquakes. ${ }^{14,15}$ It is unknown how social isolation or mood symptoms like depression or anxiety among homebound older adults were impacted by COVID-19 restrictions and associated challenges. It is critical to examine these issues as we consider the long-term impact of COVID-19 on 
the health and well-being of the homebound. Therefore, we use a nationally representative sample to assess whether COVID-19 had greater impact on social contact and mood among older adults who were homebound prior to the pandemic.

\section{METHODS}

\section{Sample/Data Set}

We used the National Health and Aging Trends Study (NHATS), a nationally representative longitudinal study of Medicare beneficiaries age 65 and over. ${ }^{16}$ Since 2011, NHATS annually surveys respondents in person on health and disability, including homebound status. In 2020 NHATS initiated a supplemental survey to capture COVID-related experiences that was fielded June-October 2020 via mail. The 2020 NHATS COVID-19 survey queries changes in contact with family and friends as well as changes in wellbeing, asking questions about contacts and mood during COVID-19 as well as prior. We included all respondents who were community-dwelling during the 2019 survey, had regional data available describing COVID-19 incidence, and completed a COVID-19 survey ( $n=3,112$; see Supplementary Appendix for details). Response rate for the COVID-19 survey was $83.5 \%$. On average, the COVID-19 survey was completed 388 days after the 2019 NHATS survey.

\section{Measures}

"Previously homebound" was broadly defined as those who either rarely or never left home or left the home at least twice/ week but reported either difficulty or receiving help to do so in 2019. ${ }^{17,18}$

The primary outcome measure was limited social contact in 2020 , operationalized as self-report of contact with family or friends during COVID-19<1/week through four communication mediums: (1) phone calls, (2) emails/texts/social media, (3) video calls, or (4)in-person visits.

Secondary outcomes captured other changes in both communication and mood during the COVID-19 pandemic that we hypothesized might vary by homebound status. These included loneliness during COVID-19 (categorized as every/ most days vs. some days, rarely, or never), increasing loneliness during COVID-19 compared to prior (via self-report of any changes: increasing, the same, or decreasing), and depression and anxiety during COVID-19 (self-reported by respondents and categorized as moderate/severe vs. mild/not at all).

In order to better contextualize communication patterns during COVID-19, we examined data on the reported frequency of contact with family/friends prior to COVID-19. This was retrospectively reported by respondents during the 2020 COVID-19 survey and defined as self-report of contact with family or friends prior to COVID-19<1/week through four communication mediums: (1) phone calls, (2) emails/texts/ social media, (3) video calls, or (4)in-person visits. Given the growth in telecommunication for both social connection and telemedicine, we additionally assessed if respondents learned a new technology during COVID-19. While homebound status was not measured by NHATS during 2020, we evaluated life space constriction (report that the respondent never left their yard or neighborhood during COVID-19). ${ }^{19}$

In order to account for regional and temporal variations in the COVID-19 pandemic that could shape both social restrictions and pandemic experience, we used publicly available COVID19 infection data that reported county-level peak incidence by day. ${ }^{20}$ We used the respondent's 2019 address to link to the county-level COVID-19 incidence. As the NHATS interview day was not available, we used the 15th (thus approximately median) day of the month of the respondent interview. We categorized county-level COVID-19 incidence rate into quintiles, with the lowest quintile ranging from 0 to 194 cases/ 100,000 persons and the highest quintile ranging from 2,539 to 18,560 cases $/ 100,000$ persons. We used peak incidence and not current incidence in order to account for how early COVID19 surges may have impacted experience.

Additional measures derived from 2019 included sex, age, race/ethnicity, self-reported income (categorized as the lowest quartile for all Medicare beneficiaries that year), if a proxy was required for survey completion, probable dementia (determined through proxy/self-reportand/or direct cognitive assessment conducted ${ }^{21}$ ), living alone, marital status, social network size or the number of people the respondent talks with about important things ( $0-1$ people, 2 people, $3+$ people), living in an assisted living facility, and residing in a metropolitan area.

\section{Analysis}

First, we conducted a bivariate comparison of the sociodemographic and COVID-experience characteristics described above between the homebound and non-homebound using a logistic regression model for each characteristic. Next, we conducted a multivariable logistic regression analysis examining the impact of previous homebound status on limited family/friend contact during COVID-19 among those with at least 1 contact/week prior to COVID-19. This included 93\% of the full sample: 2,314 non-homebound older adults and 589 homebound older adults. Given the smaller sample size for this analysis, we included variables that have been shown to be associated with being homebound, and that may also shape COVID-19 experience. Given the association of severe COVID-19 with age and the early-pandemic association of COVID-19 outbreaks with race and ethnicity as well as wealth, we included covariates for demographics (age, sex, race and ethnicity, income), as well as dementia and facility residence as these factors shape both homebound status and social outreach, and COVID-19 incidence. ${ }^{22-24}$ All analyses used Stata Version 16 and accounted for survey design and sampling approach. 
Table 1. Comparison of COVID-19 Experience of Community-Dwelling Older Adults by 2019 Homebound Status

\begin{tabular}{|c|c|c|c|}
\hline & Not homebound $(N=\mathbf{2 4 5 5})$ & Homebound $(N=657)$ & $\begin{array}{l}p- \\
\text { value }\end{array}$ \\
\hline \multicolumn{4}{|l|}{ Sociodemographics: } \\
\hline Female & $53.8 \%$ & $64.9 \%$ & 0.01 \\
\hline Age, mean(SE) & $76.6(0.1)$ & $80.4(0.4)$ & $<0.001$ \\
\hline \multicolumn{3}{|l|}{ Race } & 0.01 \\
\hline White, non-Hispanic & $83.0 \%$ & $79.7 \%$ & \\
\hline Black, non-Hispanic & $6.8 \%$ & $12.6 \%$ & \\
\hline Hispanic & $6.9 \%$ & $9.0 \%$ & \\
\hline Other & $3.3 \%$ & $4.7 \%$ & \\
\hline Lowest income quartile & $17.4 \%$ & $37.8 \%$ & $<0.001$ \\
\hline Probable dementia & $2.9 \%$ & $18.8 \%$ & $<0.001$ \\
\hline Proxy respondent & $5.4 \%$ & $28.9 \%$ & $<0.001$ \\
\hline Lives alone & $29.7 \%$ & $33.7 \%$ & 0.08 \\
\hline Married/partnered & $58.8 \%$ & $42.9 \%$ & $<0.001$ \\
\hline \multicolumn{3}{|l|}{ Social network size } & 0.10 \\
\hline $0-1$ people & $29.3 \%$ & $31.5 \%$ & \\
\hline 2 people & $27.1 \%$ & $31.6 \%$ & \\
\hline $3+$ people & $43.6 \%$ & $36.9 \%$ & \\
\hline Lives in an assisted living facility & $3.7 \%$ & $12.4 \%$ & $<0.001$ \\
\hline Resides in a metropolitan region & $82.8 \%$ & $79.1 \%$ & 0.21 \\
\hline Before COVID-19, contact with family/friends < once/week through all modalities ${ }^{1}$ & $4.0 \%$ & $9.0 \%$ & 0.001 \\
\hline \multicolumn{4}{|l|}{ COVID-19 experience: } \\
\hline \multicolumn{3}{|l|}{ Quintile of COVID-19 incidence (county-level) ${ }^{2}$} & 0.20 \\
\hline Lowest $(0-194$ cases $/ 100,000)$ & $7.1 \%$ & $5.2 \%$ & \\
\hline $2^{\text {nd }}(194-564$ cases $/ 100,000)$ & $20.8 \%$ & $20.9 \%$ & \\
\hline $3^{\text {rd }}(564-1,245$ cases $/ 100,000)$ & $29.9 \%$ & $25.6 \%$ & \\
\hline $4^{\text {th }}(1,245-2,539$ cases $/ 100,000)$ & $31.6 \%$ & $34.8 \%$ & \\
\hline Highest $(2,539-18,560$ cases $/ 100,000)$ & $10.5 \%$ & $13.5 \%$ & \\
\hline \multirow{2}{*}{\multicolumn{4}{|c|}{ During COVID-19, never left: }} \\
\hline & & & \\
\hline Yard & $1.9 \%$ & $13.0 \%$ & $<0.001$ \\
\hline Neighborhood & $10.9 \%$ & $40.2 \%$ & $<0.001$ \\
\hline Learned new technology & $30.4 \%$ & $16.3 \%$ & $<0.001$ \\
\hline \multicolumn{4}{|l|}{ During COVID-19, in contact with friends/family < once/week: } \\
\hline By phone & $11.3 \%$ & $17.4 \%$ & 0.001 \\
\hline By email & $28.4 \%$ & $54.9 \%$ & $<0.001$ \\
\hline By video & $71.1 \%$ & $77.9 \%$ & 0.01 \\
\hline In person & $57.5 \%$ & $64.6 \%$ & 0.01 \\
\hline Primary outcome: in contact < once/week; all above types & $6.5 \%$ & $13.2 \%$ & $<0.001$ \\
\hline \multicolumn{4}{|l|}{ Secondary outcomes: } \\
\hline During COVID-19, felt lonely every/most days & $7.7 \%$ & $13.2 \%$ & 0.003 \\
\hline Felt lonely more often during COVID-19 than before & $21.7 \%$ & $21.5 \%$ & 0.92 \\
\hline Moderately/severely anxious during COVID-19 & $29.6 \%$ & $26.2 \%$ & 0.20 \\
\hline Moderately/severely depressed during COVID-19 & $22.6 \%$ & $24.6 \%$ & 0.42 \\
\hline
\end{tabular}

Data source: National Health and Aging Trends Study. Homebound status and sociodemographic variables derived from 2019 survey data. COVID-19 experience variables derived from 2020 survey data. ${ }^{1}$ Defined as communication with friends/family < once/week via phone, email/text/social media, inperson, or video. ${ }^{2}$ Defined as the peak county-level incidence of COVID-19 cases at or prior to the month of the respondent interview. All proportions adjusted to account for survey design and weighting.

\section{RESULTS}

Among those who completed the COVID-19 survey and were living in the community, $16.2 \%$ were homebound in 2019. Of these previously homebound, $18.6 \%$ rarely/ never left home, $13.0 \%$ left home but never by themselves, and $68.5 \%$ left home but had difficulty doing so. Table 1 depicts complete demographic, health, and function characteristics of the cohort in 2019. The previously homebound had an average age of 80.4 years, $64.9 \%$ were female, and $18.8 \%$ had probable dementia. Compared to older adults who were not homebound prior to COVID-19, they were older, had lower income, and had higher rates of comorbidities and disability.

During COVID-19, homebound older adults were more likely to report limited family/friend contact through all communication modalities $(p<0.05$, Fig. 1). Differences were particularly pronounced for email/text/social media contact, with $54.9 \%$ of homebound reporting limited use of this modality with family/friends compared to $28.4 \%$ of nonhomebound older adults.

While the previously homebound reported more loneliness during the pandemic compared to those who were not homebound (13.2\% vs. $7.7 \%$ ), they did not experience greater increases in loneliness during COVID-19. Similarly, the homebound did not report more depression or anxiety during COVID-19. They reported greater restriction in life space (never leaving house or yard) during COVID-19 compared to those who were not previously homebound ( $13.0 \%$ vs. $1.9 \%)$. While $16.3 \%$ of homebound older adults reported learning new technology during COVID-19, 30.4\% of non-homebound older adults did. 
Phone
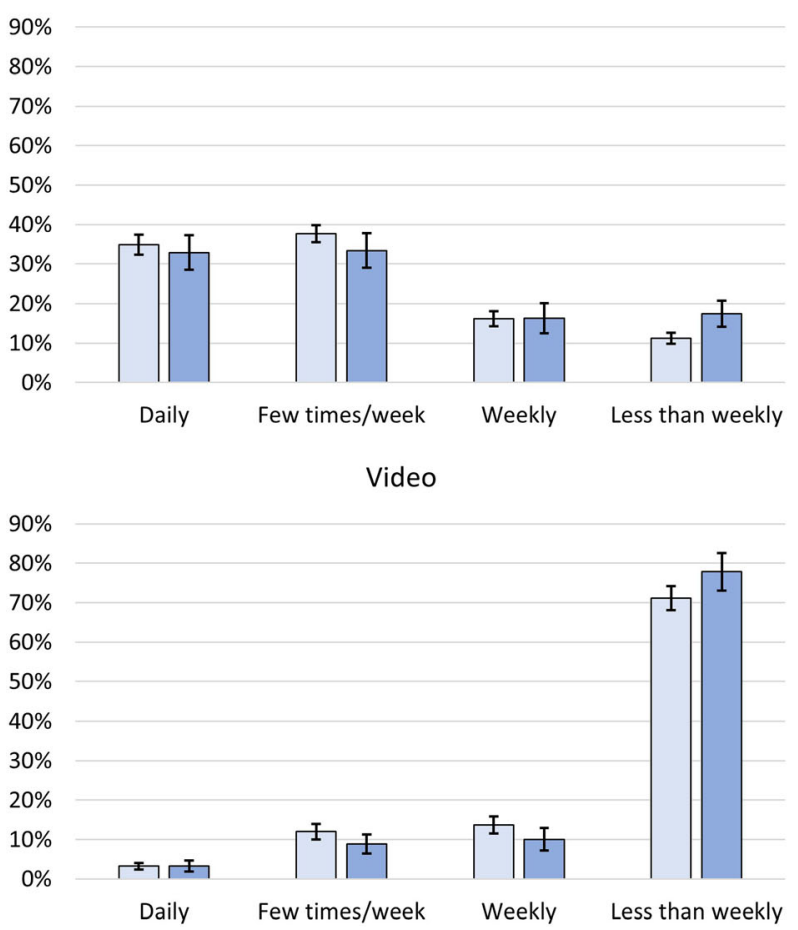

Email, text, social media
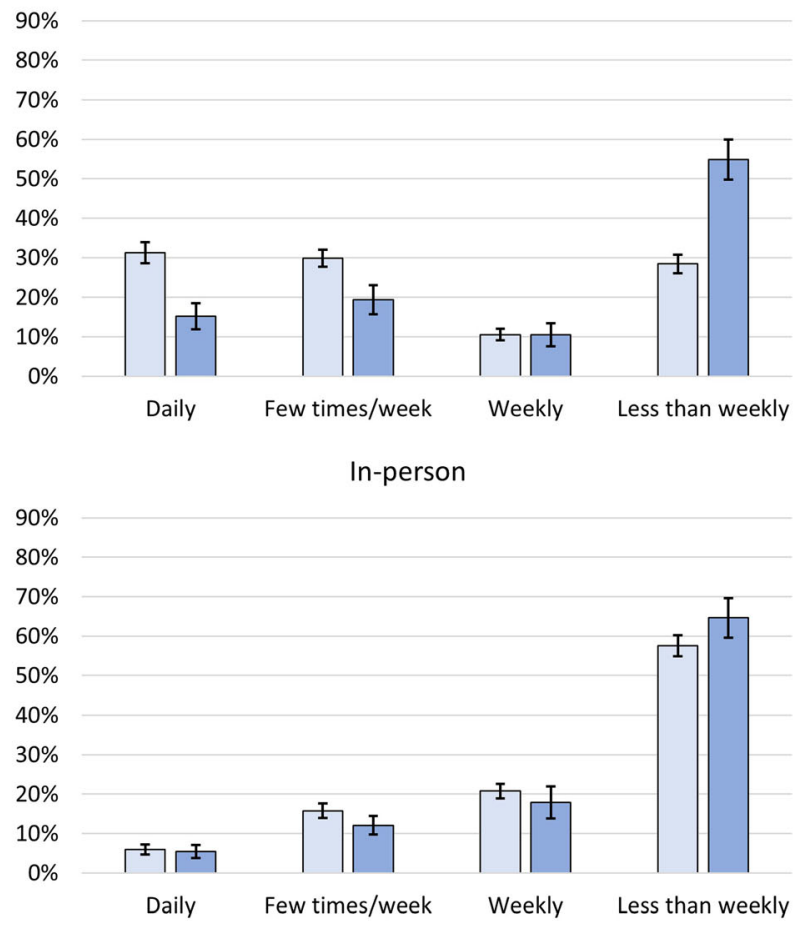

Non-homebound

Homebound

Figure 1. Rates of communication with family/friends by type during 2020 COVID-19 pandemic for previously homebound vs. non-homebound community-dwelling older adults. Legend: light blue, not homebound; dark blue, homebound.

Finally, to examine the impact of the pandemic among those without limited social contact prior to COVID-19, we limited the sample to participants with at least 1 social contact per week prior to COVID-19. In this subsample, 99 individuals $(3.5 \%)$ had limited social contact during COVID-19. In adjusted models (Table 2), the previously homebound had greater but not statistically significantly different risk $(\mathrm{OR}=1.83$; 95\% CI $0.95-$ 3.52) of limited social contact during COVID-19. Multiple characteristics associated with being homebound were also associated with risk of limited social contact: Hispanic/Latino ethnicity, probable dementia, and residing in a facility.

\section{DISCUSSION}

While the COVID-19 pandemic compelled many Americans to stay home more and become homebound, those who were homebound prior to the pandemic faced an even greater burden of social isolation. We found that previously homebound older adults were significantly more likely to have infrequent social contact across all communication mediums during the pandemic, even after excluding those with infrequent social contact prior to the pandemic. This may be least partially explained by reduced uptake of digital technology among the homebound, who emailed, texted, and used social media at lower rates and were less likely to adapt to new technology during the pandemic.
Table 2. Characteristics Associated with Odds of New Infrequent Contact with Family/Friends of Any Type During COVID-19 (<1 contact/week of any type), $N=2,773^{1}$

\begin{tabular}{lll}
\hline \hline & $\begin{array}{l}\text { Odds ratio (95\% } \\
\text { confidence interval) }\end{array}$ & $\begin{array}{l}\boldsymbol{p} \text { - } \\
\text { value }\end{array}$ \\
\hline Homebound & $1.83(0.95-3.52)$ & 0.07 \\
Age & $0.98(0.93-1.03)$ & 0.49 \\
Female & $0.55(0.34-0.89)$ & 0.02 \\
Race and ethnicity & & \\
$\quad$ White, non-Hispanic & Reference & \\
Black, non-Hispanic & $0.44(0.18-1.08)$ & 0.07 \\
Other, non-Hispanic & $1.40(0.39-4.94)$ & 0.60 \\
Hispanic & $0.20(0.04-1.00)$ & 0.05 \\
Lowest income quartile & $1.51(0.88-2.59)$ & 0.14 \\
Probable dementia & $2.19(1.18-4.07)$ & 0.01 \\
Lives alone & $0.59(0.27-1.28)$ & 0.18 \\
Lives in a facility & $2.89(1.06-7.92)$ & 0.04 \\
Lives in a metropolitan region & $0.95(0.59-1.52)$ & 0.82 \\
Highest county-level incidence & $1.18(0.65-2.15)$ & 0.57 \\
of COVID-19 & & \\
\hline
\end{tabular}

Data source: National Health and Aging Trends Study. ${ }^{1}$ Cohort restricted to those who did not report infrequent social contacts prior to COVID-19, in order to measure odds of new infrequent outreach. Homebound status and sociodemographic variables derived from 2019 survey data. COVID-19 experience variables derived from 2020 survey data. ${ }^{2}$ Defined as top two quartiles of county-level incidence of COVID19 cases at or prior to the month of the respondent interview, equivalent to $\geq 1,245$ cases/100,000 persons. Reference group is those in the lower two quartiles of county-level incidence of COVID-19 cases. ${ }^{3}$ Reference group was those in the 2nd-4th income quartiles. Models adjusted to account for survey design and weighting. 
Our findings of increased social isolation must be understood in the context of the growing body of work on the experience of homebound older adults. This population has a high burden of functional and cognitive disability and thus must rely on caregivers and external systems of care to remain in the community. ${ }^{25}$ Despite their relatively high isolation at baseline, our research demonstrates that COVID-19 disproportionately reduced their social contact, which may have impacted their ability to access care, material supports, and health care. In a post-COVID19 landscape where digital communication and telehealth are even more prevalent, the lower rates of using email, text, and social media and learning new technologies among the homebound may continue to cut them off from needed resources. ${ }^{26}$

Unexpectedly, and despite limited social interaction and higher rates of loneliness among the homebound, homebound older adults did not report greater COVID-19-relatedincreases in depression, anxiety, or loneliness compared to nonhomebound older adults. This may demonstrate the resilience of homebound older adults in coping with a higher degree of isolation over time. ${ }^{27,28}$ Alternatively, these surveys may have been conducted too early in the pandemic (predominantly during the summer of 2020) for the psychological impact of increased isolation to have fully impacted the homebound population. Finally, we do not have nuanced psychological data on other mood symptoms, nor do we have the sample size within the scope of this study on homebound older adults to elucidate factors contributing to risks of mood symptoms. Further research will need to assess the psychological sequelae of COVID-19 over time among older adults including the homebound, in terms of longer-term effects and protective factors such as social contacts inside and outside the home.

Although a strength of our work is that we examine a national sample prospectively, we are challenged to address variation in the pandemic experience by place and time. Fortunately, the NHATS survey will continue to annually assess the experiences of the cohort, so future research will be able to assess changes in experience over the course of the pandemic. While we draw on county-level data on COVID-19 incidence rates at the time the respondent was surveyed, local variation in both the severity of restrictions and perceptions of the risks of the pandemic may not be captured by county-level COVID19 incidence. In addition, measures of COVID-19 incidence in many locations have been underestimated due to limited testing availability. While we are able to capture the homebound status of this cohort in 2019, the 2020 COVID-19 survey did not ask about homebound status so we are unable to assess if homebound status changed during COVID-19. We also do not know how household structures changed: some families may have proactively moved together to reduce isolation at the onset of the pandemic. Finally, we do not have complete data on the potential consequences of the diminished social contact experienced by the homebound during COVID-19, such as unmet caregiving needs or difficulty obtaining food. Further survey and qualitative data will be important to fully explore the experience of homebound adults.
We must ensure that homebound persons have access to the care that they need to remain safely in the community, which is even more challenging in a global pandemic. We are only now beginning to measure the impacts of social isolation, which we find has disproportionately impacted the homebound during COVID-19. For bedside clinicians, identifying social isolation $^{29}$ and opening conversations with patients and families is important as we all come to better understand the impact of the pandemic on older adults. As we move forward, it will be important to design, test, and disseminate new models to help support the social connection, and mental and physical health of those who have experienced months of isolation and restricted activity during the pandemic. Homebound older adults already have especially complex needs due to medical, financial, and social vulnerability coupled with a high degree of functional and cognitive disability and medical illness. While other populations have expanded their use of digital technology to meet their social and health needs during the COVID-19 pandemic (for example, telemedicine use and online COVID-19 testing and vaccination sign-ups), we find that the homebound may not be able to adapt in this way. Finding models to understand and overcome barriers to digital technologies and telehealth for the homebound is critical to helping them maintain health and wellness during the COVID-19 pandemic and future public health crises.

Corresponding Author: Claire K. Ankuda, MD, MPH; Brookdale Department of Geriatrics and Palliative Medicine, Icahn School of Medicine, New York, NY, USA (e-mail: Claire.ankuda@mssm.edu).

Supplementary Information The online version contains supplementary material available at https://doi.org/10.1007/s11606-02107361-9.

Author Contribution Study concept and design: C.K.A., K.O., A.K., K.H., J.R.; methods: C.K.A., K.O., A.K., K.H., J.R.; data collection: C.K.A., K.O.; analysis: C.K.A.; K.O.; preparation of paper: C.K.A., K.O., A.K., K.H., J.R.

Funding Dr. Ankuda is funded by the National Institute on Aging (K76AG064427), Dr. Harrison is funded by the National Institute on Aging (K01AG059831, P01AG066605), Dr. Ornstein is funded by the National Institute on Aging (R01AG060967; P30AG028741), and Dr. Reckrey is funded by the National Institute on Aging (K23AG066930). The sponsor had no role in design, methods, subject recruitment, data collection, analysis, and preparation of the paper.

Declarations: The authors declare that they do not have a conflict of interest.

\section{REFERENCES}

1. Ornstein KA, Leff B, Covinsky KE, et al.Epidemiology of the homebound population in the United States. JAMA Intern Med. 2015;175(7):1180-1186.

2. Hayes S SC, McCarthy D, Radley DC, Abrams S, Shah T, Anderson G.High-Need, High-Cost Patients: Who Are They and How Do They Use Health Care? A Population-Based Comparison of Demographics, Health Care Use, and Expenditures. 2016. August 29, 2016. 
3. Wajnberg A, Ornstein $\mathbf{K}$, Zhang $\mathbf{M}$, Smith KL, Soriano T. Symptom burden in chronically ill homebound individuals. $J$ Am Geriatr Soc. Jan 2013;61(1):126-31. https://doi.org/10.1111/jgs. 12038

4. Soones T, Federman A, Leff B, Siu AL, Ornstein K.Two-year mortality in homebound older adults: an analysis of the National Health and Aging Trends Study. J Am Geriatr Soc. Jan 2017;65(1):123-129. https://doi. org/10.1111/jgs. 14467

5. Jacobs JM, Hammerman-Rozenberg A, Stessman J. Frequency of leaving the house and mortality from age 70 to 95 . J Am Geriatr Soc. Nov 22 2017. https://doi.org/10.1111/jgs.15148

6. Desai NR, Smith KL, Boal J. The positive financial contribution of homebased primary care programs: the case of the Mount Sinai Visiting Doctors. JAmGeriatrSoc. 2008;56(4):744-749. NOT IN FILE.

7. Negron-Blanco L, de Pedro-Cuesta J, Almazan J, Rodriguez-Blazquez C, Franco E, Damian J. Prevalence of and factors associated with homebound status among adults in urban and rural Spanish populations. BMC Public Health. Jul 15 2016;16:574. https://doi.org/10. 1186/s12889-016-3270-Z

8. Nicholson NR. A review of social isolation: an important but underassessed condition in older adults. J Prim Prev. Jun 2012;33(2-3):13752. https://doi.org/10.1007/s10935-012-0271-2

9. Cohen-Mansfield $\mathbf{J}$, Shmotkin $\mathbf{D}$, Hazan $\mathbf{H}$. The effect of homebound status on older persons. J Am Geriatr Soc. Dec 2010;58(12):2358-62. https://doi.org/10.1111/j.1532-5415.2010.03172.x

10. Choi NG, Marti CN, Bruce ML, Hegel MT. Depression in homebound older adults: problem-solving therapy and personal and social resourcefulness. Behav Ther. Sep 2013;44(3):489-500. https://doi.org/10.1016/ j.beth.2013.04.002

11. Stokes EK, Zambrano LD, Anderson KN, et al. Coronavirus disease 2019 case surveillance-United States, January 22-May 30, 2020. Morb Mortal Weekly Rep. 2020;69(24):759.

12. Franzosa E, Gorbenko K, Brody AA, et al. "At Home, with Care": Lessons from New York City Home-based Primary Care Practices Managing COVID-19. J Am Geriatr Soc. Nov 12 2020;https://doi.org/ $10.1111 /$ jgs. 16952

13. Sterling MR, Tseng E, Poon A, et al. Experiences of home health care workers in New York City during the coronavirus disease 2019 pandemic: a qualitative analysis. JAMA Intern Med. Aug 4 2020;180(11):1453-9. https://doi.org/10.1001/jamainternmed.2020.3930

14. Hirai H, Kondo $\mathbf{N}$, Sasaki R, et al. Distance to retail stores and risk of being homebound among older adults in a city severely affected by the 2011 Great East Japan Earthquake. Age Ageing 2014;44(3):478-484. https://doi.org/10.1093/ageing/aful46

15. Malik S, Lee DC, Doran KM, et al. Vulnerability of older adults in disasters: emergency department utilization by geriatric patients after Hurricane Sandy. Disaster Med Public Health Prep. 2018;12(2):184- 193. https://doi.org/10.1017/dmp.2017.44

16. Freedman VA, Kasper JD. Cohort Profile: The National Health and Aging Trends Study (NHATS). Int J Epidemiol. Aug 1 2019;48(4):1044-1045g. https://doi.org/10.1093/ije/dyz109

17. Ornstein KA, Leff B, Covinsky KE, et al. Epidemiology of the homebound population in the United States. JAMA Intern Med.
Jul 2015; 175(7):1180-6. https://doi.org/10.1001/jamainternmed.2015. 1849

18. Ornstein KA, Garrido MM, Bollens-Lund E, et al. The association between income and incident homebound status among older Medicare beneficiaries. J Am Geriatr Soc. Aug 10 2020. https://doi.org/10.1111/ jgs. 16715

19. Stalvey BT, Owsley C, Sloane ME, Ball K. The Life Space Questionnaire: a measure of the extent of mobility of older adults. J Appl Gerontol. 1999/12/01 1999;18(4):460-478. https://doi.org/10.1177/ 073346489901800404

20. Dong E, Du H, Gardner L. An interactive web-based dashboard to track COVID-19 in real time. Lancet Infect Dis. May 2020;20(5):533-534. https://doi.org/10.1016/s1473-3099(20)30120-1

21. Kasper JD, Freedman VA, Spillman B.Classification of persons by dementia status in the National Health and Aging Trends Study. Technical Paper \#5. . 2013. May be accessed at www.nhats.org

22. Bassett MT, Chen JT, Krieger N. Working Paper Series: The unequal toll of COVID-19 mortality by age in the United States: quantifying racial/ ethnic disparities. June 28, 2020, Updated June 12, 2020. https://cdn1. sph.harvard.edu/wp-content/uploads/sites/1266/2020/06/20_Bassett-Chen-Krieger_COVID-19_plus_age_working-paper_0612_Vol-19_No3_with-cover.pdf. Accessed 1/10/2022

23. Banerjee A, Pasea L, Harris S, et al. Estimating excess 1-year mortality associated with the COVID-19 pandemic according to underlying conditions and age: a population-based cohort study. The Lancet. 2020;395(10238):1715-1725. https://doi.org/10.1016/s0140-6736(20) 30854-0

24. Gross CP, Essien UR, Pasha S, Gross JR, Wang SY, Nunez-Smith M. Racial and ethnic disparities in population-level Covid-19 mortality. J Gen Intern Med. Oct 2020;35(10):3097-3099. https://doi.org/10.1007/ s11606-020-06081-w

25. Reckrey JM, Federman AD, Bollens-Lund E, Morrison RS, Ornstein KA.Homebound status and the critical role of caregiving support. J Aging Soc Policy. Jun 27 2019:1-14. https://doi.org/10.1080/08959420.2019.1628625

26. Kalicki AV, Moody KA, Franzosa E, Gliatto PM, Ornstein KA. Barriers to telehealth access among homebound older adults. J Am Geriatr Soc. Apr 13 2021. https://doi.org/10.1111/jgs.17163

27. Kotwal AA, Holt-Lunstad J, Newmark RL, et al. Social isolation and loneliness among San Francisco Bay Area older adults during the COVID-19shelter-in-place orders. J Am Geriatr Soc. Jan 2021;69(1):2029. https://doi.org/10.1111/jgs. 16865

28. Luchetti M, Lee JH, Aschwanden D, et al. The trajectory of loneliness in response to COVID-19. Am Psychol. Oct 2020;75(7):897-908. https:// doi.org/10.1037/amp0000690

29. Russell DW. UCLA Loneliness Scale (Version 3): Reliability, validity, and factor structure. J Pers Assess. 1996/02/01 1996;66(1):20-40. https:// doi.org/10.1207/s15327752jpa6601_2

Publisher's Note Springer Nature remains neutral with regard to jurisdictional claims in published maps and institutional affiliations. 